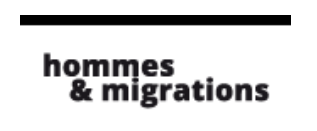

\section{Hommes \& migrations}

Revue française de référence sur les dynamiques

migratoires

$1317-1318$ | 2017

L'Europe en mouvement

\title{
Mémoire de migrations entre Italie et France
}

\section{Stéphane Mourlane et Matteo Sanfilippo}

\section{CpenEdition \\ Journals}

\section{Édition électronique}

URL : http://journals.openedition.org/hommesmigrations/3865

DOI : 10.4000/hommesmigrations.3865

ISSN : 2262-3353

\section{Éditeur}

Musée national de l'histoire de l'immigration

\section{Édition imprimée}

Date de publication : 1 avril 2017

Pagination : 25-35

ISBN : 978-2-919040-38-4

ISSN : $1142-852 X$

\section{Référence électronique}

Stéphane Mourlane et Matteo Sanfilippo, « Mémoire de migrations entre Italie et France », Hommes \& migrations [En ligne], 1317-1318 | 2017, mis en ligne le 01 avril 2020, consulté le 04 janvier 2020. URL : http://journals.openedition.org/hommesmigrations/3865; DOI : 10.4000/hommesmigrations.3865 


\title{
MÉMOIRE DE MIGRATIONS ENTRE ITALIE ET FRANCE
}

Par STÉPHANE MOURLANE, historien, maître de conférences en histoire contemporaine, Aix-Marseille Université-CNRS, TELEMMe, et MATTEO SANFILIPPO, historien, professeur en histoire moderne, Università della Tuscia, et directeur du Centro Studi Emigrazione de Rome.

\author{
De part et d'autres des Alpes, la gestion de la mémoire \\ de l'immigration connaît des formes diversifiées. Si l'héroïsation \\ des émigrants partis dès la fin du XIXe siècle a court en Italie, \\ le pays fait face au défi d'enrichir son histoire de celles \\ des nouveaux immigrants qui viennent s'y installer. Du côté \\ français, la mémoire de la présence italienne a gagné \\ en visibilité grâce à de prestigieux ambassadeurs du monde \\ de la culture et du sport. Mais elle demeure surtout l'apanage \\ des descendants des migrants eux-mêmes. La conscience d'une \\ mémoire partagée entre la France et I'Italie reste en devenir.
}

Dans l'histoire de l'Europe contemporaine, l'Italie est l'un des principaux pays d'émigration, tandis que la France figure parmi les premiers pays d'immigration. L'émigration chez l'un a nourri l'immigration chez l'autre. Les migrations transalpines sont anciennes. À partir du Moyen Âge, clercs, marchands, banquiers, artistes, mais aussi colporteurs et paysans de ce pays qui n'est encore qu'une " expression géographique » trouvent en France une terre d'accueil. Le mouvement prend de l'ampleur à partir du milieu du XIX ${ }^{e}$ siècle alors que la France manque de main-d'œuvre et que l'Italie affiche un trop-plein que son économie ne parvient pas à absorber, en plus de connaître de fortes tensions politiques conduisant à l'exil. À l'heure de la Grande émigration, entre 1876 et 1915, un peu plus de 6 millions d'Italiens, sur les 14 millions d'émigrants, partent vers des pays européens (les autres ont pour destination les Amériques). La France arrive en tête des pays européens de destination en accueillant 1,6 million d'Italiens. Et si on n'en recense que 420000 à la veille de la guerre, c'est que la fluidité de la circulation est grande. Au cours de l'entre-deux-guerres, la France devient même le principal pays d'accueil de l'émigration italienne devant les États-Unis qui établissent une politique de quotas au début des années 1920. Ils sont encore 1,6 million à traverser les Alpes. 800000 Italiens sont recensés en 1931 - sans doute un million en incluant les saisonniers et les clandestins -, soit 7 \% de la population hexagonale. Après la Seconde Guerre mondiale, si les flux restent importants jusqu'aux années 1960 (près de 700 000), d'autres pays européens, comme l'Allemagne, la Suisse et la GrandeBretagne, deviennent plus attractifs. Pendant près d'un siècle, les Italiens ont représenté la première nationalité étrangère en France.

Historiens italiens et français ont produit depuis une trentaine d'années de nombreux travaux, d'un côté, sur l'émigration, de l'autre, sur l'immigration, et plus récemment en prenant en compte le phénomène migratoire transalpin dans une perspective plus globale, plus transnationale. Si l'his- 
toire est désormais bien connue, on est surpris d'observer que la mémoire de cette migration, de part et d'autre des Alpes, rencontre plus de difficultés à s'affirmer. Parfois oublié ou méconnu, souvent déformé au prisme du misérabilisme ou de l'idéalisation, ce passé migratoire trouve sa place dans la mémoire collective des deux pays selon des modalités qui mêlent à la fois ressorts historiques et enjeux contemporains. Dans un regard croisé, entre Italie et France, nous voudrions ici proposer des premiers éléments d'analyse.

\section{La formation d'un mythe en Italie}

Si la mémoire de l'émigration est longtemps restée essentiellement dans la sphère individuelle ou familiale, diverses manifestations dans l'espace public ont toutefois contribué à forger un imaginaire collectif. Dès la naissance de l'État unitaire italien, la question migratoire et sa représentation tiennent une place importante dans le débat public. Pendant la période des gouvernements libéraux (1861-1922), la presse, l'Église catholique et les représentants des partis politiques interviennent à plusieurs reprises sur le sujet. Tandis que l'Église postule la nécessité d'aider ceux qui partent, la presse et les partis, surtout ceux au gouvernement, tendent à décrire l'émigration croissante comme un danger, une source d'affaiblissement pour le pays. Pour tous, il apparaît essentiel de maintenir, voire même de révéler en cette période post-unitaire où " les Italiens restent à faire », l'italianité des migrants. Leur attachement nostalgique à l'Italie est souligné en 1911 lors de l'inauguration de l'Autel de la Patrie, le Vittoriano, bâti à Rome pour commémorer le cinquantenaire de l'Unité italienne ${ }^{1}$. Auparavant, des expositions se sont efforcées de présenter positivement l'émigration comme le triomphe du génie italien dans le monde ${ }^{2}$.

La formation de communautés italiennes à l'étranger est en outre conçue comme une forme de " colonisation ». Le Congrès des Italiens à l'étranger est ainsi organisé en 1911 à l'Institut colonial italien. Les « colonies » italiennes aux Amériques doivent contribuer au rayonnement économique dans le Nouveau Monde tandis que les « colonies » italiennes en Afrique du Nord permettent de pénétrer des espaces déjà sous le contrôle d'autres pays colonisateurs ${ }^{3}$. Le régime fasciste développe davantage le lien entre émigration et expansionnisme. Pour Mussolini, «l'Italie ne fournira plus d'émigrants vers le monde. Il s'agit désormais d'envoyer le génie glorieux de sa race à travers le monde ». Il cherche à encadrer et orienter les flux vers l'Afrique, l'Égée et les îles de la Dalmatie, ainsi que vers des territoires intérieurs encore à exploiter, comme les marécages du Latium et de la Sardaigne ${ }^{4}$. Les Italiens constituent un "peuple de voyageurs, d'explorateurs », comme il est écrit sur le fronton du Palais du travail construit pour l'Exposition universelle de Rome prévue en 1942.

Après la Seconde Guerre mondiale, le gouvernement républicain ne rompt pas radicalement avec cette mythologie de l'émigration. Il n'est pas fait obstruction aux départs, tant l'émigration constitue une soupape de sécurité pour apaiser les tensions socio-politiques ${ }^{5}$. De plus, il poursuit la politique fasciste de migrations intérieures et octroie

1. Une exposition est consacrée à l'émigration italienne tandis que figure sur un pilier l'inscription d'un hommage des "Italiens à l'étranger à la mère-patrie ». 2. Notamment l'Italian-American Exhibition à Gênes (1892), le pavillon des Italiens à l'étranger à l'Exposition nationale de Turin (1898) ou encore l'Exposition des Italiens à l'étranger à Milan en relation avec l'Exposition internationale (1906). Voir Mark Choate, Emigrant Nation: The Making of Italy Abroad, Cambridge (Mass.), Harvard University Press, 2008. 3. Stefano Pelaggi, Il colonialismo popolare. L'emigrazione e la tentazione espansionistica italiana in America Latina, Rome, Edizioni Nuova Cultura, 2015 ; Marco Soresina, « Italian emigration policy during the Great Migration Age, 1888-1919: the interaction of emigration and foreign policy ", in Journal of Modern Italian Studies, vol. 21, $n^{\circ}$ 5, 2016 , pp. 723-746. 4. Stefano Gallo, Il Commissariato per le migrazioni e la colonizzazione interna (1930-1940). Per una storia della politica migratoria del fascismo, Foligno, Editoriale Umbra, 2015 ; « II villaggio “Luigi Razza” a Làgosta/Lastovo. Mobilità e conflitti nella colonizzazione fascista di un'isola dalmata (1934-1943) ", in Michele Colucci, Michele Nani (dir.), Lavoro mobile. Migranti, organizzazioni, conflitti (XVIII-XX secolo), Palerme, New Digital Frontiers, 2015, pp. 147-180. 5. Michele Colucci, Lavoro in movimento. L'emigrazione italiana in Europa, 1945-57, Rome, Donzelli, 2008 ; Michele Colucci, "La politica migratoria italiana ", in Zilli Ilaria (dir.), Un ponte sull'oceano. Migrazioni e rapporti economici fra Italia e Argentina dall'unità a oggi, Naples, CNRISSM, 2012, pp. 45-61. 
la pleine liberté de mouvement aux travailleurs et le transfert de la main-d'œuvre méridionale vers la capitale et le triangle industriel. Dès ce moment, la mobilité intérieure devint un élément essentiel du modèle migratoire italien ${ }^{6}$. Même le retour de certains émigrés se caractérise par le choix, en Italie, d'un lieu différent de celui de départ.

\section{Mémoires de l'émigration et immigration en Italie}

À partir des années 1970, le modèle migratoire italien se modifie avec l'arrivée de migrants dans la Péninsule. Le mouvement se renforce dans les années 19907. La fermeture progressive des frontières des pays du Nord de l'Europe, la croissance économique ainsi que la proximité géographique avec des régions à fort potentiel migratoire (Maghreb, Balkans) ont conduit à une croissance des flux dans les années 1980. En 2010, plus de 4,2 millions d'étrangers sont installés dans la Péninsule. L'ampleur soudaine de ces flux ébranle la société italienne. L'arrivée massive de migrants irréguliers dans les ports méridionaux en provenance d'Albanie au début des années 1990, ainsi que, plus récemment, les naufrages à répétition d'embarcations de clandestins suscitent une vive émotion au sein de l'opinion publique ${ }^{8}$. Les incidents xénophobes survenus depuis se multiplient. Les injures, les coups et les assassinats, qui touchent principalement les extracommunautari, interpellent nombre d'Italiens. Le racisme se retrouve aussi dans le discours politique. Les " dangers de l'immigration » deviennent le cheval de bataille des mouvements de la droite localiste, telle la Ligue Nord, et ensuite des formations populistes, telles que les Cinq Étoiles. Au mythe de l'invasion se superpose un discours liant immigration clandestine, insécurité et criminalité.

Cette " crise migratoire ", qui touche l'Europe et qui place l'Italie en première ligne, est concomitante d'une nouvelle émigration italienne. En 2015, l'Italie enregistre 107000 émigrés, dont plus qu'un tiers est âgé de moins de 34 ans.

Cette évolution n'a fait que relancer un mécanisme triangulaire fait d'émigration, d'immigration et de mobilité intérieure ${ }^{9}$. C'est dans ce contexte que l'on assiste à un mouvement de résurgence mémorielle concernant l'émigration des années 1860-1960.

Le lien entre cette émigration et l'immigration que connaît l'Italie est fréquemment explicité. Ainsi en est-il de l'essai de Gian Antonio Stella, L'orda. Quando gli albanesi eravamo noi (2003). Pour le journaliste du Corriere della Sera, il convient de rappeler que, "dans ces années de confrontation aux "hordes" d'immigrés en Italie et de montée de la xénophobie, que nous avons été les immigrés des autres ${ }^{10}$ ". La patrimonialisation de l'émigration au travers de musées intègre l'immigration récente dans une continuité historique et dans une perspective pédagogique et morale face à la xénophobie. Le comité scientifique qui a travaillé à l'élaboration du musée de Rome n'a certes pas été entendu dans

La patrimonialisation de l'émigration au travers de musées intègre l'immigration récente dans une continuité historique et dans une perspective pédagogique et morale face à la xénophobie. sa proposition d'un musée des migrations. Le gouvernement de Silvio Berlusconi, soucieux de distinguer la "bonne " émigration italienne de la "mauvaise » immigration étrangère, a préféré un musée national de l'émigration italienne. Il n'en reste pas moins que ce musée propose dans sa dernière section consacrée à la période de 1977 à nos jours, à côté d'un panorama de la réalité actuelle des Italiens dans le monde, une évocation du "monde accueilli par l'Italie". Lors de son inauguration, le 23 octobre 2009, le président de la République, Giorgio Napolitano, 
Salles de l'exposition Ciao Italia, avril 2017. Photo : Anne Volery. @ PaLAIS DE LA PoRTE DoréE.

ancien dirigeant du Parti communiste, déclare en outre: "Noublions pas que nous avons été des émigrants », une formule mise en exergue le lendemain dans de nombreux journaux. D'autres musées de l'émigration suivent ce modèle. Le musée de la mer et de la navigation de Gênes l'affiche même ouvertement en intitulant son troisième étage, inauguré en 2011, "Mémoires et migrations », et non pas seulement émigration. La dernière section est consacrée à l'immigration, présentant une barcone utilisée pour traverser le canal de Sicile avec des témoignages photographiques et filmées de sauvetage en mer, de débarquements et même de naufrages. Depuis juillet 2013, le Museo Narrante dell'Emigrazione, La nave della Sila, inauguré en 2005 à Camigliatello Silano en Calabre, donne à voir « d'une part, l'expérience italienne dans le monde, d'autre part, l'expérience étrangère en Italie ${ }^{11}$ ». Les discriminations et la xénophobie, dont les Italiens ont été les victimes, sont systématiquement abordées et font aussi référence en creux à la situation des immigrés en Italie. 
Salles de l'exposition Ciao Italia, avril 2017. Photo : Anne Volery. ๑) Palais de la Porte Dorée.

Le passage de l'Italie d'un pays d'émigration à un pays d'immigration a également suscité un intérêt et un nouvel essor de l'historiographie de l'émigration $^{12}$. Dans les années 2000, des centaines de volumes sont publiées sur l'émigration, l'immigration et la mobilité intérieure en Italie ${ }^{13}$. L'heure est à l'étude des "migrations ». Des revues spécialisées, telles que Studi Emigrazione (fondée en 1964), Altreitalie (1989) et Archivio storico dell'emigrazione italiana (2005), jouent un rôle essentiel dans la stimulation et la diffusion des connaissances au sein de la communauté scientifique. L'attention se porte notamment sur le mécanisme des réseaux en comparant émigration, immigration et mobilité intérieure ${ }^{14}$.

\section{Mémoires tragiques de l'émigration italienne}

Toutefois, ces publications de spécialistes n'ont qu'un faible écho dans l'opinion au sein de laquelle la mémoire de l'émigration demeure dominée par le dolorisme et l'héroïsme. Les musées, les expositions ${ }^{15}$, les livres ou encore les recherches subventionnées par les régions ou par l'État exaltent un passé de souffrance. Les migrants y apparaissent comme des aventuriers dont le sacrifice a permis à l'Italie de rattraper son retard économique. Sont organisées notamment des manifestations pour 
commémorer le centenaire ou le cinquantenaire de tragédies dans les mines d'Europe et des Amériques: Monongah aux États-Unis, Mattmark en Suisse et surtout, peut-être, Marcinelle $e^{16}$. Ce drame apparaît dans les médias comme le symbole du sacrifice des migrants italiens. En novembre 2003, Porta a Porta, populaire talk-show de la télévision publique nationale, propose une émission avec la participation du dernier survivant avant diffusion d'un téléfilm produit dans le cadre d'un projet de la RAI sur la " Mémoire du XX $X^{e}$ siècle ». La commémoration en 2006 du cinquantenaire du drame de Marcinelle bénéficie d'une large couverture médiatique, notamment à la télévision avec la diffusion d'un documentaire. La RAI propose par ailleurs des soirées de variété, des documentaires ou encore des mini-séries : Carramba, che sorpresa! (19961998), Carramba, che fortuna! (1998-1999), Poveri noi (1999), Le ali della vita (2000), Come l'America (2001) ou encore La terra del ritorno (2004).

La littérature joue aussi sur la corde sensible du drame. Toute une série de romans tragiques sont publiés comme Vita de Melania G. Mazzucco, qui obtient le Prix Strega en 2003, Quando Dio ballava il tango de Laura Pariani en 2002, présenté par l'éditeur comme un "roman de l'émigration et de la mémoire ", Pane amaro. Un immigrato italiano in America, un roman publié par Elena Gianini Beltottin, inspiré de l'histoire vraie de son père qui émigre en 1919 aux États-Unis ou encore Il giorno prima della felicità d'Erri De Luca en 2009. Le cinéma s'inscrit dans la même veine dramatique à l'image de Nuovomondo d'Emanuele Crialese, qui remporte le Lion d'argent au festival de Venise en
2006 et qui, sur un mode lyrique, retrace l'itinéraire d'une famille du Mezzogiorno ${ }^{17}$ jusqu'en Amérique où elle doit faire face à des désillusions. La chanson de Giammaria Testa, Da questa parte del mare, en 2006, fournit une autre forme d'écho à cette mémoire tragique. La quotidienneté de l'émigration semble échapper à cette mémoire. Celleci trouve à s'exprimer seulement de manière plus confidentielle dans le nouveau cinéma et le nouveau roman des immigrés ${ }^{18}$.

\section{Un intérêt renouvelé pour l'émigration}

Fort de cette attention croissante pour les émigrants italiens du passé, le gouvernement lance toute une série d'initiatives au début du XXI ${ }^{\mathrm{e}}$ siècle en vue de mieux insérer l'émigration au sein de la communauté nationale.

Jusqu'alors, la question du rapport à l'émigration et de sa mémoire est surtout du ressort des régions ou des provinces. Ce sont elles qui se préoccupent d'abord de retisser le lien avec ceux qui sont partis à l'étranger, pour des raisons économiques et commerciales. Elles soutiennent les associations régionales qui se développent dans les pays d'immigration. Les collectivités locales apportent souvent leur concours à l'essor de très nombreux musées de l'émigration dans les régions ${ }^{19}$.

Au niveau national, la loi pour le vote des Italiens de l'extérieur est un tournant marquant l'intérêt renforcé porté à l'émigration. L'accès à la nationalité facilité pour les descendants d'émigrants le

\footnotetext{
15. C'est en 1988 que se tient la première grande exposition nationale à Rome, L'Italia fuori d'Italia : immagini di emigrazione ; en 2003, est organisée au Vittoriano de Rome une autre exposition qui préfigure le musée national de l'émigration italienne, Tante Patrie una Patria - L'identità italiana nel mondo attraverso l'emigrazione. 16. Le 8 août 1956, au charbonnage du Bois du Cazier en Belgique, un wagonnet mal engagé dans sa remontée vers la surface sectionne des fils électriques et provoque un court-circuit. Un incendie s'ensuit : 262 ouvriers, dont 136 Italiens, périssent. Voir Norberto Lombardi (dir.), Monongah 1907. Una tragedia dimenticata, Rome, MAE, 2007 ; Toni Ricciardi, Morire a Mattmark. L'ultima tragedia dell'emigrazione italiana, Rome, Donzelli, 2015 et Marcinelle, 1956. Quando la vita valeva meno del carbone, Rome, Donzelli, 2016. 17. Le Mezzogiorno (en français " le Midi ") désigne l’ensemble des régions péninsulaire et insulaire qui correspondent au sud de l'Italie. Sa définition est différente de celle de l'Italie méridionale qui ne comprend pas les régions insulaires. 18. Emma Bond, Guido Bonsaver, Federico Faloppa (dir.), Destination Italy: Representing Migration in Contemporary Media and Narrative, Oxford, Peter Lang, 2015. 19. On peut en citer notamment dans le Piémont, Museo Regionale de l'Emigrazione Piemontesi nel Mondo, Museo dell'Emigrante di Roasio ; en Ombrie, Museo Regionale dell'Emigrazione Piero Conti ; en Toscane, Museo Paolo Cresci, Museo dell'Emigrazione della Gente Toscana ; en Émilie-Romagne, Museo dell'emigrante di Tarsogno; dans le Frioul, Museo dell'emigrazione di cavasso nuovo ; en Sicile, la Rete dei Musei Siciliani dell'Emigrazione.
} 
confirme. Il suffit désormais d'avoir des grandsparents nés en Italie ${ }^{20}$. À l'occasion de la Prima conferenza degli italiani nel mondo en 2000, le gouvernement organise plusieurs rencontres avec des parlementaires, des hommes d'affaires et des journalistes d'origine italienne d'autres pays.

La commémoration des 150 ans de l'unité italienne, en 2011, est aussi l'occasion d'asseoir et de valoriser l'émigration dans la mémoire et l'histoire nationales. Le Musée national de l'émigration italienne de Rome en fournit l'exemple le plus éclatant. Son implantation à l'Autel de la Patrie, monument en hommage à Victor-Emmanuel II et célébrant l'unité italienne, renforce plus encore une forte dimension symbolique en faveur de l'intégration de l'émigration à la mémoire officielle nationale. Lors de son discours inaugural, le président de la République Gingio Napolitano souligne la nécessité " d'insérer légitimement la contribution de l'émigration italienne au lent et progressif processus d'unification nationale ». Pour Lorenzo Prencipe, président du comité scientifique du musée, il s'agit de «déchirer le voile de silence qui a accompagné l'émigration italienne au cours de ces 150 ans, comme si ceux qui étaient partis ne valaient rien pour l'Italie ${ }^{21}$ ".

\section{Dissiper l'invisibilité de l'immigration italienne}

En France, une chape de silence a également recouvert pendant un certain temps ces Italiens venus en nombre ${ }^{22}$. Alors qu'ils représentent encore la première nationalité étrangère dans l'Hexagone dans les années 1960, ils sont invisibles dans l'espace public ${ }^{23}$. Les médias n'accordent que peu d'intérêt à l'immigration italienne, portant leur attention sur l'immigration maghrébine ${ }^{24}$. Au cinéma, le film d'Yves Allegret, La meilleure part, sorti en 1955, et Mademoiselle de Tony Richardson en 1966 sont parmi les derniers à mettre en scène des ouvriers italiens. La situation tranche avec le passé, et ce qu'autant plus qu’à la xénophobie s'est substituée une appréciation plutôt favorable. Une enquête de l'Ined place, en 1951, les Italiens au quatrième rang de sympathie après les Belges, les Suisses, les Hollandais et avant les Espagnols et les Polonais ${ }^{25}$. S'il subsiste encore quelques préjugés $^{26}$, l'opinion est principalement fondée sur le sentiment traditionnel d'affinité culturelle qui distingue les Italiens des populations d'immigration plus récente dont on juge l'altérité plus accuAu début des années 1980, le chanteur italo-belge Claude Barzotti le proclame fièrement dans une chanson à grand succès en France : "Je suis Rital et je le reste ». Au même moment, en 1983, I'historien Pierre Milza, fils d'immigrés de la plaine du Pô, publie Voyage en Ritalie, première grande synthèse de l'histoire de l'immigration transalpine en France. sée. La bienveillance dont bénéficient les Italiens et leur invisibilité médiatique expliquent la difficulté de voir émerger une mémoire de l'immigration italienne.

Dans les années 1970, quelques jalons servent de révélateur à cette mémoire. L’un des premiers marqueurs est la chanson "L'Italien » interprétée en 1971 par Serge Reggiani ${ }^{27}$. Fils d'un antifasciste de Reggio d'Émilie arrivé en France en 1930, le chanteur évoque le retour au pays d'un migrant à l'itinéraire chaotique. Cette chanson au ton très grave donne à entendre, à la fois en français et en italien, toute la difficulté de la migration et l'attachement du migrant à son pays d'origine. Quelques années plus tard, en 1978, François

20. Guido Tintori (dir.), Il voto degli altri, Turin, Rosenberg \& Sellier, 2012. 21. Lorenzo Prencipe, "La storia d'un grande esodo : Il museo nazionale dell' emigrazione italiana ", in Rapporto Italiani nel Mondo, 2010, Todi, Tau Editrice. 22. Stéphane Mourlane, Céline Regnard, "Invisibility and memory. Italian immigration in France during the second half of the 2 oth century ", in Lisa Anteby-Yemini, Virginie Baby-Collin, Sylvie Mazzella, Stéphane Mourlane, Cédric Parizot, Céline Regnard, Pierre Sintès (dir.), Borders, Mobilities and Migrations. Perspectives from the Mediterranean, Bruxelles, Peter Lang, 2014, pp. 267-287. 23. Yvan Gastaut, L'immigration et l'opinion en France sous la Ve République, Paris, Seuil, 2000. 24. Stéphane Mourlane, "Une certaine idée de l'Italie. Attitudes et politique français. 1958-1969 ", thèse de doctorat en histoire contemporaine, Université de Nice, 2002. URL : https://halshs.archives-ouvertes.fr/tel-01115304. 25. Alain Girard, Jean Stoetzel, Français et immigré. L'attitude française. L'adaptation des Italiens et des Polonais, Paris, Ined, Travaux et documents, 1954. 26. Stéphane Mourlane, "Que reste-til des préjugés? L'opinion française et l'immigration italienne dans les années 1950-1960 ", in Migrations Société, $\mathrm{n}^{\circ} 109,2007$, pp. 133-145. 27. Stéphane Mourlane, "Yves Montand, Serge Reggiani, c'est nous... les Italiens ? ", in Volume !, n², 2015, pp. 21-30. 
Giacomina L. (au centre), née à Raveo (Udine, Frioul) en 1932. Arrivée en France pour la première fois en 1955, puis rentrée dans son village natal, elle vit avec son mari Pietro à Montreuil. @ VERONICA MECCHIA.

Cavanna raconte dans Les Ritals son enfance dans les années 1930 à Nogent-sur-Marne où réside une importante communauté italienne. Le livre reçoit un bon accueil auprès de la critique et est adapté pour un film à la télévision. L'appellation "ritals », employée pour désigner depuis les années 1950, de manière certes péjorative mais bienveillante, les immigrés italiens, apparaît désormais assumée et même revendiquée par ces derniers. Au début des années 1980, le chanteur italo-belge Claude Barzotti le proclame fièrement dans une chanson à grand succès en France : « Je suis Rital et je le reste ${ }^{28} »$. Au même moment, en 1983, l'historien Pierre Milza, fils d'immigrés de la plaine du Pô, publie Voyage en Ritalie, première grande synthèse de l'histoire de l'immigration transalpine en France. L'ouvrage, qui intègre une part d'ego-histoire de l'auteur, historicise auprès d'un public plus large que la communauté scientifique la mémoire de l'immigration italienne et, par-là, lui donne toute sa légitimité.

La mémoire de l'immigration italienne ressurgit également autour d'une grande figure sportive : Michel Platini, meilleur joueur de football français des années 1970-198029. Lors de son transfert au club de la Juventus à Turin, la presse française souligne qu'il s'agit d'un « retour dans le pays de ses parents" (en fait de ses grands-parents). Pour autant, Platini apparaît comme un symbole de l'intégration. Et, justement, la résurgence de 
la mémoire de l'immigration italienne entre les années 1970 et 1990 doit beaucoup à la valorisation de la capacité à l'intégration des Italiens.

\section{Mémoires et culture italienne en France}

À la fin des années 1990, on assiste à une forte demande sociale qui se traduit par l'évocation fréquente du « devoir de mémoire » qui s'exprime à plusieurs niveaux de la société ${ }^{30}$. Les descendants de migrants entendent dans ce contexte à la fois redécouvrir et obtenir reconnaissance de leur histoire.

Les Français d'origine étrangère se retrouvent autour de "lieux de mémoire " afin de commémorer le souvenir des temps de la migration. Pour ceux qui sont issus de l'immigration italienne, il n'est pas anodin que le temps de la commémoration corresponde à la fin du processus migratoire. La question de l'intégration étant digérée, les individus d'une même appartenance peuvent regarder le passé avec apaisement et rendre hommage à ceux qui ont rendu leur présence en France possible. L'appartenance à la nation n'étant plus un enjeu, la mémoire de l'immigration italienne trouve, à l'échelle régionale, un cadre propice à son expression, notamment à travers des associations de plus en plus nombreuses. Les cours de langue, conférences, voyages en Italie et autres activités proposées par les Institut culturels italiens à Paris, Lyon, Marseille et Grenoble ainsi que par les 27 comités de Dante Alighieri dans l'Hexagone, rencontrent un public large, formé souvent de descendants de migrants désireux de mieux connaître le pays de leurs parents, grands-parents ou arrière-grandsparents. Les associations sont très actives dans les jumelages entre villes française et italienne qui établissent des liens transnationaux entre communautés d'accueil et de départ. Ils apparaissent souvent comme une manière originale de commémorer un réseau migratoire ${ }^{31}$. Le développement d'Internet et des réseaux sociaux favorise la dynamique associative et communautaire. En Îlede-France, le Forum des associations franco-italiennes se présente par exemple comme un portail et un agenda d'activités ${ }^{32}$. Parmi ces associations, L'Italie à Paris dispose d'un site Internet très consulté mettant à disposition une vaste palette d'informations culturelles (musique, théâtre, cinéma, livres, expositions, conférences, cours d'italien, gastronomie...) $)^{33}$.

On note également un nombre croissant d'expositions sur l'immigration italienne. Elles s'inscrivent L'appartenance à la nation n'étant plus un enjeu, la mémoire de l'immigration italienne trouve, à l'échelle régionale, un cadre propice à son expression, notamment à travers des associations de plus en plus nombreuses. parfois dans le cadre d'un projet mémoriel plus vaste. Ainsi, l'exposition organisée en 2012 à Paris « Sur les traces de l'immigration italienne » est l'aboutissement d'un projet lancé en 2010 visant à recueillir des témoignages et des chants au sein de la communauté italienne de l'Est parisien. Ces expositions ont souvent une dimension locale comme "Un air d'Italie » au Musée dauphinois de Grenoble en $2011^{34}$. De mars à septembre 2017, l'exposition "Ciao Italia " au Musée national de l'histoire de l'immigration à Paris "rend compte pour la première fois à l'échelle nationale, de l'histoire de l'immigration italienne en France, qui reste à ce jour la plus importante de l'histoire française ${ }^{35}$ ».

Parmi les manifestations culturelles mettant en scène la mémoire de l'immigration italienne, une place grandissante est donnée au spectacle. La démarche artistique a une vocation mémorielle, mais aussi pédagogique pour ne pas dire politique, au moment où en France l'immigration est souvent stigmatisée autour d'un débat sur l'identité 
nationale voulu par le président Sarkozy ${ }^{36}$. Ainsi la pièce de théâtre Sale août, montée en 2010 à partir des travaux de l'historien Gérard Noiriel, sur le massacre d'Aigues-Mortes en $1893^{37}$. Comme l'écrit Libération, "Pogrom, procès scandaleux, mémoire étouffée, le massacre d'Aigues-Mortes ne figure pas parmi les pages les plus glorieuses de l'histoire hexagonale 38 ». Le journal y voit « une pièce contemporaine ", notamment au regard de la passivité de la population face au drame. La mémoire de l'immigration italienne sert ici, dans une perspective militante, de parabole à l'actualité migratoire. Il en est de même du spectacle, dont le titre fait écho aux formules employées en Italie : "Italiens. Quand les immigrés c'était nous ». Présenté par le groupe Incanto à la Maison de la mutualité

à Paris en novembre 2016,

Le cinéma et la télévision

apparaissent plus en retrait

dans ce retour de mémoire.

Au cinéma, les films mettant

en scène l'immigration italienne sont rares. il se présente comme "un hymne à la solidarité et la tolérance ». À l'origine de cette initiative se trouve Rocco Femia, rédacteur en chef de la revue Radici et directeur de la maison d'édition Editalia, qui œuvre à une meilleure connaissance de la culture italienne en France et de l'histoire de l'immigration transalpine. La Voce, " magazine des Italiens de France », et Focus In qui entend "valoriser tout ce qui est italien en France " s'inscrivent aussi dans cette perspective, partagée également par des stations de radios locales qui proposent des « émissions italiennes ${ }^{39}$ ".

Le cinéma et la télévision apparaissent plus en retrait dans ce retour de mémoire. Au cinéma, les films mettant en scène l'immigration italienne sont rares. Dans son deuxième film, Rouge Midi, en 1985, Robert Guédiguian raconte l'histoire de Maggiorna, une Calabraise installée dans le quartier de l'Estaque dans les années 1920. Marseille est également le cadre du film Bella Ciao en 2001 qui évoque la migration de communistes italiens qui s'y installent en 1932 alors qu'ils rêvent d'Amé- rique. Dans un autre registre cinématographique, le festival du film italien de Villerupt, créé en 1976, est devenu un événement culturel majeur. Il participe de la diffusion auprès de l'ensemble de la population régionale d'un imaginaire italien positif qui rejaillit sur les personnes qui s'en réclament. Le sculpteur italo-lorrain Amilcar Zannoni, auteur du trophée l'Amilcar remis lors du palmarès du festival, évoque ainsi : "Un cinéma humainement engagé, apprécié d'une population pouvant être fière d'avoir joué un rôle essentiel dans l'histoire culturelle de la Lorraine ${ }^{40}$. »

Le festival de Villerupt donne lieu en 1982 à l'un des rares documentaires sur l'immigration italienne : L'Anniversaire de Thomas : Villerupt, ville du fer. Le film d'Axel Clevenot et Gérard Noiriel, Buongiorno della Francia, produit par l'INA et FR3, est le seul à proposer, en 1986, une histoire globale de cette immigration en France. Sur le petit écran, quelques fictions portent la trace de l'immigration italienne. La mini-série Fabio Montale diffusée par TF1 en janvier 2012 met en scène un fils de Napolitain. Elle est tirée de la trilogie romanesque Total Kheops, Chourmo, Solea de Jean-Claude Izzo, né d'un père italien arrivé en 1928 de la région de Salerne à Marseille.

Preuve sans doute que cette mémoire de l'immigration italienne touche toutes les générations et tous les milieux culturels, elle trouve à s'exprimer dans le rap par la voix de Philippe Fragione, plus connu sous le nom d'Akhenaton, au sein du groupe de rap marseillais IAM. En 1995, dans son premier album solo Métèque et mat, il fait référence à ses origines napolitaines. En 2001, dans Paese, un titre en hommage à l'Italie dans l'album Sol Invictus, il chante : « J'viens d'là où parler avec les mains, c'est vital. »

\section{Conclusion}

Tant en Italie qu'en France, la résurgence de la mémoire transalpine s'inscrit dans un temps 
Le Pas de Centocroci en Italie qui sépare la Val di Vara en Émilie-Romagne, en s'ouvrant sur l'Apennin ligure, relie la commune de Varese Ligure (La Spezia) avec celle de Albareto (Parme).

Beaucoup d'immigrés en France sont originaires de l'Apennin tosco-émilien. @ Veronica Mecchia.

« troublé par l'inquiétant spectacle que donnent le trop de mémoire ici, le trop d'oubli ailleurs, pour ne rien dire de linfluence des commémorations et des abus de mémoire et d'oubli ${ }^{41} »$. Dans les deux pays, le rapport quelque peu chaotique au passé migratoire est en grande partie déterminé par les soubresauts politiques et sociaux causés par les nouvelles migrations. Il manifeste une perspective commune distinguant une « bonne » émigration/immigration, celle des Italiens, d'une « mauvaise » immigration extra-européenne. Pour autant, ces mémoires ne semblent pas jouer un rôle de resserrement des liens entre les « sœurs latines » tant elles s'expriment de manière autonome l'une de l'autre. Il est vrai qu'en Italie les diverses formes d'expressions mémorielles privilégient l'émigration transocéanique. En outre, le processus de patrimonialisation s'essouffle par manque de moyens : le Musée national de l'émigration italienne a fermé ses portes tandis que le projet d' «Ellis Island italien ${ }^{42}$ » à Gênes peine à se concrétiser. En France, l'intérêt demeure confiné à des cercles culturels restreints souvent en lien avec les descendants de migrants. On s'intéresse toujours plus à l'Italie comme "terre des arts et du patrimoine ». Du chemin reste donc à parcourir pour l'établissement d'une mémoire partagée, transnationale, même si quelques initiatives émergent. La collaboration entre historiens italiens et français, que cet article incarne, doit pouvoir y contribuer. 